\title{
A Probabilistic Fixed Point Result Using Altering Distance Functions
}

\author{
Claudia Zaharia ${ }^{1}$ and Nataša Ćirović ${ }^{2}$ \\ ${ }^{1}$ Department of Mathematics, West University of Timişoara, Bulevardul V. Pârvan 4, 300223 Timişoara, Romania \\ ${ }^{2}$ Department of Mathematics, Faculty of Electrical Engineering, University of Belgrade, Bulevar Kralja Aleksandra 74, \\ 11000 Belgrade, Serbia
}

Correspondence should be addressed to Claudia Zaharia; claudia.zaharia@e-uvt.ro

Received 26 February 2015; Accepted 3 August 2015

Academic Editor: Richard I. Avery

Copyright ( 92015 C. Zaharia and N. Ćirović. This is an open access article distributed under the Creative Commons Attribution License, which permits unrestricted use, distribution, and reproduction in any medium, provided the original work is properly cited.

We prove a general fixed point theorem in Menger spaces for mappings satisfying a contractive condition of Ćirić type, formulated by means of altering distance functions. Thus, we extend some recent results of Choudhury and Das, Miheț, and Babačev and also clarify some aspects regarding a theorem of Choudhury, Das, and Dutta.

\section{Introduction and Preliminaries}

In [1], Menger introduced the concept of probabilistic metric space as a generalization of metric spaces, in which the distance between points is expressed by means of distribution functions. This idea has made probabilistic metric spaces suitable for modeling phenomena when the uncertainty regarding measurements is assumed as inherent to the measuring process, as, for instance, in the investigation of certain physical quantities and physiological thresholds [2]. Probabilistic metric space theory has become a very active field of research. In particular, fixed point theory in probabilistic structures has found relevant applications in studying the existence and uniqueness of solutions of random equations [3], as well as algorithm complexity analysis $[4,5]$, and convergence analysis for stochastic optimization algorithms [6].

In the present paper, we establish a fixed point result for probabilistic contractions of Ćirić type, with the contractive condition stated by means of an altering distance function. Our theorem is obtained under very weak hypotheses, and thus it generalizes or improves several known results [7-10]. We also discuss the connections with a related theorem given by Choudhury et al. in [11], in order to explain the role of our assumptions.

We begin by recalling some fundamental concepts of probabilistic metric space theory. For a comprehensive exposition on this topic we refer the reader to the monographs $[2,3]$.

Definition 1. A triangular norm (or $t$-norm) is a mapping $T$ : $[0,1] \times[0,1] \rightarrow[0,1]$ which is associative, commutative, and nondecreasing in each variable and satisfies $T(x, 1)=x$ for all $x \in[0,1]$.

Some basic examples are $T_{M}(x, y)=\min (x, y)$, $T_{P}(x, y)=x y$, and $T_{L}(x, y)=\max (x+y-1,0)$ (the minimum, product, and Łukasiewicz $t$-norm, resp.). Another important class is that of $t$-norms of Hadžić type [12], that is, $t$-norms whose family of iterates $\left\{T^{n}(x)\right\}_{n}$ defined by $T^{0}(x)=$ $1, T^{n}(x)=T\left(T^{n-1}(x), x\right) \forall n \geq 1$, is equicontinuous at $x=1$.

We will denote by $\mathscr{D}^{+}$the set of functions $F: \mathbb{R} \rightarrow[0,1]$ which are nondecreasing and left continuous on $\mathbb{R}$, such that $F(0)=0$ and $\lim _{t \rightarrow \infty} F(t)=1$.

Definition 2. A Menger space is a triple $(X, F, T)$ where $X$ is a nonempty set, $F$ is a mapping from $X \times X$ to $\mathscr{D}^{+}$, and $T$ is a $t$-norm, such that the following conditions are satisfied:

(i) $F_{x y}(t)=1$ for all $t>0$ iff $x=y$.

(ii) $F_{x y}(t)=F_{y x}(t)$, for all $x, y \in X, t>0$.

(iii) $F_{x y}(t+s) \geq T\left(F_{x z}(t), F_{z y}(s)\right)$, for all $x, y, z \in X, t, s>$ 0 . 

$\left.F_{x y}.\right)$

(Here and in the following, $F(x, y)$ will be denoted by

Let $(X, F, T)$ be a Menger space such that $\sup _{x \in(0,1)}$ $T(x, x)=1$. The family $\{U(\varepsilon, \lambda)\}_{\mathcal{E}>0, \lambda \in(0,1)}$, where

$$
U(\varepsilon, \lambda)=\left\{(x, y) \in X \times X: F_{x y}(\varepsilon)>1-\lambda\right\},
$$

is a base for a Hausdorff uniformity on $X$, named strong uniformity. The corresponding, strong topology on $X$ is introduced by the family of neighbourhoods of $x \in X \mathcal{N}_{x}=$ $\left\{N_{x}(\varepsilon, \lambda)\right\}_{\varepsilon>0, \lambda \in(0,1)}$, where $N_{x}(\varepsilon, \lambda)=\left\{y \in X: F_{x y}(\varepsilon)>1-\lambda\right\}$, and this topology is metrizable [2].

Definition 3. Let $(X, F, T)$ be a Menger space. A sequence $\left(x_{n}\right)_{n}$ in $X$ is said to be

(i) Cauchy if, for any $\varepsilon>0, \lambda \in(0,1)$, there exists $n_{0} \in \mathbb{N}$ such that $F_{x_{n} x_{m}}(\varepsilon)>1-\lambda$, for all $m, n \geq n_{0}$;

(ii) convergent to $x \in X$ if, for any $\varepsilon>0, \lambda \in(0,1)$, there exists $n_{0} \in \mathbb{N}$ such that $F_{x_{n} x}(\varepsilon)>1-\lambda$ for all $n \geq n_{0}$.

$X$ is said to be complete if every Cauchy sequence $\left(x_{n}\right)_{n} \subset X$ is convergent in $X$.

If the $t$-norm $T$ is continuous, and the sequences $\left(x_{n}\right)_{n}$, $\left(y_{n}\right)_{n} \subset X$ converge, respectively, to $x$ and $y \in X$, then $F_{x_{n} y_{n}}(t)$ converges to $F_{x y}(t)$, for each continuity point $t$ of $F_{x y}$ [2].

Definition 4. Given a set $A \subset X$, the probabilistic diameter of $A$ is the mapping $D_{A}: \mathbb{R} \rightarrow[0,1]$ defined by

$$
D_{A}(t)= \begin{cases}\sup _{s<t} \inf _{x, y \in A} F_{x y}(s), & t>0 \\ 0, & t \leq 0 .\end{cases}
$$

$A$ is said to be probabilistically bounded if $D_{A} \in \mathscr{D}^{+}$.

The notion of contraction in a Menger space was introduced by Sehgal in [13].

Definition 5 (see [13]). Let $(X, F, T)$ be a Menger space. A mapping $f: X \rightarrow X$ is said to be a probabilistic contraction (or Sehgal contraction) if there exists $c \in(0,1)$ such that

$$
F_{f(x) f(y)}(t) \geq F_{x y}\left(\frac{t}{c}\right), \quad \forall x, y \in X, t>0 .
$$

Many significant contributions to the development of fixed point theory in probabilistic structures can be found in monograph [14]. It should be pointed out that the triangular norm by which the space is endowed plays a key role in the existence of fixed points of probabilistic contractions. It was shown by Radu [15] that the largest class of continuous $t$ norms $T$ with the property that every Sehgal contraction on a complete Menger space $(X, F, T)$ has a unique fixed point is that of $t$-norms of Hadžić type.

The idea of using altering distance functions in order to obtain more general contractive conditions first appears in [16], in the setting of metric spaces. The corresponding concept of generalized probabilistic contraction was introduced by Choudhury and Das in [8] as follows.
Definition 6 (see [8]). A mapping $\varphi:[0, \infty) \rightarrow[0, \infty)$ is said to belong to the class $\Phi$ if it satisfies

(i) $\varphi(t)=0$ iff $t=0$;

(ii) $\varphi$ is strictly increasing and $\lim _{t \rightarrow \infty} \varphi(t)=\infty$;

(iii) $\varphi$ is continuous at $t=0$ and left continuous on $(0, \infty)$.

The mappings $\varphi \in \Phi$ will be called altering distance functions.

Definition 7 (cf. [8]). Let $(X, F, T)$ be a Menger space. The mapping $f: X \rightarrow X$ is said to be a generalized probabilistic contraction of Choudhury-Das type if there exist $\varphi \in \Phi$ and $c \in(0,1)$ such that

$$
F_{f(x) f(y)}(\varphi(t)) \geq F_{x y}\left(\varphi\left(\frac{t}{c}\right)\right), \quad \forall x, y \in X, t>0 .
$$

It was proved in [8] that such contractions on a complete Menger space endowed with the strongest $t$-norm $T_{M}$ have a unique fixed point. The result was subsequently generalized by Miheţ [9] for the case of arbitrary continuous $t$-norms, under the supplementary assumption that the orbit of the mapping $f$ at some $x \in X$ is probabilistically bounded.

Our aim is to prove a fixed point result for mappings satisfying the more general contractive condition

$$
\begin{gathered}
F_{f(x) f(y)}(\varphi(t)) \geq \min \left(F_{x y}\left(\varphi\left(\frac{t}{c}\right)\right), F_{x f(x)}\left(\varphi\left(\frac{t}{c}\right)\right),\right. \\
\left.F_{y f(y)}\left(\varphi\left(\frac{t}{c}\right)\right)\right), \quad \forall x, y \in X, t>0,
\end{gathered}
$$

for some $\varphi \in \Phi$ and $c \in(0,1)$.

\section{Main Results}

In order to prove our results, we will need the following lemma from [10].

Lemma 8 (see $[10])$. Let $(X, F, T)$ be a Menger space; $\varphi \in \Phi$ and $c \in(0,1)$. If $x, y \in X$ are such that

$$
F_{x y}(\varphi(t)) \leq F_{x y}\left(\varphi\left(\frac{t}{c}\right)\right), \quad \forall t>0,
$$

then $x=y$.

For each $x \in X$, we will denote by $O(f, x)$ the orbit of the mapping $f$ at $x$; that is, $O(f, x)=\left\{f^{n}(x): n \in \mathbb{N}\right\}$.

Theorem 9. Let $(X, F, T)$ be a complete Menger space with $T$ a continuous t-norm. Suppose $f: X \rightarrow X$ is a mapping satisfying the contractive condition (5), for some $\varphi \in \Phi$ and $c \in$ $(0,1)$. If there exists $x \in X$ such that $O(f, x)$ is probabilistically bounded, then $f$ has a unique fixed point in $X$.

Proof. Let $x$ be as in the statement of the theorem. We will show that the sequence $\left(x_{n}\right)_{n}, x_{n}=f^{n}(x)$, is Cauchy. 
From (5) it follows that

$$
\begin{aligned}
& F_{x_{n} x_{n+1}}(\varphi(t)) \\
& \quad \geq \min \left(F_{x_{n-1} x_{n}}\left(\varphi\left(\frac{t}{c}\right)\right), F_{x_{n} x_{n+1}}\left(\varphi\left(\frac{t}{c}\right)\right)\right),
\end{aligned}
$$

$\forall t>0$.

By replacing $t$ with $t / c$ above we get

$$
\begin{aligned}
& F_{x_{n} x_{n+1}}\left(\varphi\left(\frac{t}{c}\right)\right) \\
& \quad \geq \min \left(F_{x_{n-1} x_{n}}\left(\varphi\left(\frac{t}{c^{2}}\right)\right), F_{x_{n} x_{n+1}}\left(\varphi\left(\frac{t}{c^{2}}\right)\right)\right),
\end{aligned}
$$

$\forall t>0$.

Therefore

$$
\begin{gathered}
F_{x_{n} x_{n+1}}(\varphi(t)) \geq \min \left(F_{x_{n-1} x_{n}}\left(\varphi\left(\frac{t}{c}\right)\right),\right. \\
\left.F_{x_{n-1} x_{n}}\left(\varphi\left(\frac{t}{c^{2}}\right)\right), F_{x_{n} x_{n+1}}\left(\varphi\left(\frac{t}{c^{2}}\right)\right)\right),
\end{gathered}
$$

and, inductively,

$$
\begin{gathered}
F_{x_{n} x_{n+1}}(\varphi(t)) \geq \min \left(F_{x_{n-1} x_{n}}\left(\varphi\left(\frac{t}{c}\right)\right),\right. \\
F_{x_{n-1} x_{n}}\left(\varphi\left(\frac{t}{c^{2}}\right)\right), \ldots, F_{x_{n-1} x_{n}}\left(\varphi\left(\frac{t}{c^{p}}\right)\right), \\
\left.F_{x_{n} x_{n+1}}\left(\varphi\left(\frac{t}{c^{p}}\right)\right)\right),
\end{gathered}
$$

for all $t>0$ and for any positive integer $p$. Since $\varphi$ is strictly increasing, we obtain

$$
\begin{aligned}
& F_{x_{n} x_{n+1}}(\varphi(t)) \\
& \quad \geq \min \left(F_{x_{n-1} x_{n}}\left(\varphi\left(\frac{t}{c}\right)\right), F_{x_{n} x_{n+1}}\left(\varphi\left(\frac{t}{c^{p}}\right)\right)\right),
\end{aligned}
$$

$\forall t>0$,

for all $p$. By letting $p \rightarrow \infty$ it follows that

$$
F_{x_{n} x_{n+1}}(\varphi(t)) \geq F_{x_{n-1} x_{n}}\left(\varphi\left(\frac{t}{c}\right)\right), \quad \forall t>0 .
$$

Consequently, for all $n \in \mathbb{N}$ and $t>0$,

$$
F_{x_{n} x_{n+1}}(\varphi(t)) \geq F_{x_{0} x_{1}}\left(\varphi\left(\frac{t}{c^{n}}\right)\right) \stackrel{n \rightarrow \infty}{\longrightarrow} 1 .
$$

Next, let $m$ be a positive integer. We prove by induction on $n$ that

$$
F_{x_{n} x_{n+m}}\left(\varphi\left(c^{n} t\right)\right) \geq \min \left(F_{x_{0} x_{1}}(\varphi(t)), F_{x_{0} x_{m}}(\varphi(t))\right),
$$$$
\forall t>0 \text {, }
$$

for all $n \in \mathbb{N}$. The case $n=0$ is immediate. Suppose now that inequality (14) holds for some $n \in \mathbb{N}$. Then

$$
\begin{gathered}
F_{x_{n+1} x_{n+m+1}}\left(\varphi\left(c^{n+1} t\right)\right)=F_{f\left(x_{n}\right) f\left(x_{n+m}\right)}\left(\varphi\left(c^{n+1} t\right)\right) \\
\geq \min \left(F_{x_{n} x_{n+m}}\left(\varphi\left(c^{n} t\right)\right), F_{x_{n} x_{n+1}}\left(\varphi\left(c^{n} t\right)\right),\right. \\
\left.F_{x_{n+m} x_{n+m+1}}\left(\varphi\left(c^{n} t\right)\right)\right) \geq \min \left(F_{x_{0} x_{1}}(\varphi(t)),\right. \\
\left.F_{x_{0} x_{m}}(\varphi(t)), F_{x_{0} x_{1}}(\varphi(t)), F_{x_{0} x_{1}}\left(\varphi\left(\frac{t}{c^{m}}\right)\right)\right),
\end{gathered}
$$

for all $t>0$. By the monotonicity of $\varphi$, it follows that $F_{x_{0} x_{1}}\left(\varphi\left(t / c^{m}\right)\right) \geq F_{x_{0} x_{1}}(\varphi(t))$, for all $t>0$, whence

$$
\begin{aligned}
& F_{x_{n+1} x_{n+m+1}}\left(\varphi\left(c^{n+1} t\right)\right) \\
& \quad \geq \min \left(F_{x_{0} x_{1}}(\varphi(t)), F_{x_{0} x_{m}}(\varphi(t))\right), \quad \forall t>0 .
\end{aligned}
$$

As such, we conclude that inequality (14) holds for all $m, n \in \mathbb{N}$, or equivalently

$$
\begin{aligned}
& F_{x_{n} x_{n+m}}(\varphi(t)) \\
& \quad \geq \min \left(F_{x_{0} x_{1}}\left(\varphi\left(\frac{t}{c^{n}}\right)\right), F_{x_{0} x_{m}}\left(\varphi\left(\frac{t}{c^{n}}\right)\right)\right),
\end{aligned}
$$

$\forall m, n \in \mathbb{N}, t>0$.

Now, let $\varepsilon>0$ and $\lambda \in(0,1)$. Given that $\varphi$ is continuous at 0 , there exists $t>0$ with $\varphi(t)<\varepsilon$. Also, since $D_{\mathrm{O}(f, x)} \in \mathscr{D}_{+}$, there exists $n_{0} \in \mathbb{N}$ such that $D_{\mathrm{O}(f, x)}\left(\varphi\left(t / c^{n}\right)\right)>1-\lambda$, for all $n \geq n_{0}$.

Thus,

$$
\begin{aligned}
F_{x_{n} x_{n+m}}(\varepsilon) & \geq F_{x_{n} x_{n+m}}(\varphi(t)) \\
& \geq \min \left(F_{x_{0} x_{1}}\left(\varphi\left(\frac{t}{c^{n}}\right)\right), F_{x_{0} x_{m}}\left(\varphi\left(\frac{t}{c^{n}}\right)\right)\right) \\
& \geq D_{O(f, x)}\left(\varphi\left(\frac{t}{c^{n}}\right)\right)>1-\lambda,
\end{aligned}
$$

for all $n \geq n_{0}, m \in \mathbb{N}$, and therefore $\left(x_{n}\right)_{n}$ is a Cauchy sequence. Accordingly, there exists $x^{*} \in X, x^{*}=\lim _{n \rightarrow \infty} x_{n}$.

Next, we will prove that $x^{*}$ is a fixed point of $f$. Specifically, we will show that

$$
F_{x^{*} f\left(x^{*}\right)}(\varphi(t)) \geq F_{x^{*} f\left(x^{*}\right)}\left(\varphi\left(\frac{t}{c}\right)\right),
$$

for all $t>0$.

By the contractive condition (5),

$$
\begin{gathered}
F_{x_{n+1} f\left(x^{*}\right)}(\varphi(t)) \geq \min \left(F_{x_{n} x_{n+1}}\left(\varphi\left(\frac{t}{c}\right)\right),\right. \\
\left.F_{x^{*} f\left(x^{*}\right)}\left(\varphi\left(\frac{t}{c}\right)\right), F_{x_{n} x^{*}}\left(\varphi\left(\frac{t}{c}\right)\right)\right),
\end{gathered}
$$

for all $n \in \mathbb{N}$ and $t>0$. If $t$ is such that $F_{x^{*} f\left(x^{*}\right)}$ is continuous at $\varphi(t)$, then (19) follows by letting $n \rightarrow \infty$ in the above inequality and taking into account relation (13). If $F_{x^{*}} f\left(x^{*}\right)$ 
is not continuous at $\varphi(t)$, let $\left(t_{m}\right)_{m}$ be a strictly increasing sequence converging to $t$ such that $F_{x^{*} f\left(x^{*}\right)}$ is continuous at $\varphi\left(t_{m}\right)$, for all $m \in \mathbb{N}$. As above, we infer that $F_{x^{*} f\left(x^{*}\right)}\left(\varphi\left(t_{m}\right)\right) \geq$ $F_{x^{*} f\left(x^{*}\right)}\left(\varphi\left(t_{m} / c\right)\right), \forall m \in \mathbb{N}$, whence, for $m \rightarrow \infty$, we obtain (19). By Lemma 8 we conclude that $x^{*}=f\left(x^{*}\right)$.

Finally, we prove that $x^{*}$ is the only fixed point of $f$ in $X$. To that end, let $y \in X$ be such that $f(y)=y$. Then, using (5), we get

$$
\begin{gathered}
F_{x^{*} y}(\varphi(t)) \geq \min \left(F_{x^{*} y}\left(\varphi\left(\frac{t}{c}\right)\right), F_{x^{*} x^{*}}\left(\varphi\left(\frac{t}{c}\right)\right),\right. \\
\left.F_{y y}\left(\varphi\left(\frac{t}{c}\right)\right)\right)=F_{x^{*} y}\left(\varphi\left(\frac{t}{c}\right)\right), \quad \forall t>0 .
\end{gathered}
$$

Once again, by Lemma 8 , it follows that $y=x^{*}$.

Corollary 10. If $(X, F, T)$ is a complete Menger space with $T$ a continuous $t$-norm of Hadžić type, and $f: X \rightarrow X$ satisfies condition (5) for some $c \in(0,1)$ and some $\varphi \in \Phi$ such that $\lim _{t \rightarrow \infty}(\varphi(t)-\varphi(c t))=\infty$, then $f$ has a unique fixed point in $X$.

Proof. We will show that, for every $x \in X, O(f, x)$ is probabilistically bounded. To do so, let $x \in X$ be arbitrary and define $\left(x_{n}\right)_{n}$ by $x_{n}=f^{n}(x)$ for all $n \geq 0$. We will prove by induction on $n$ that

$$
F_{x_{0} x_{n}}(\varphi(t)) \geq T^{n}\left(F_{x_{0} x_{1}}(\varphi(t)-\varphi(c t))\right), \quad \forall t>0,
$$

for all $n \geq 1$. The case $n=1$ is trivial. Suppose now that the relation holds for some $n \geq 1$. Then

$$
\begin{aligned}
F_{x_{0} x_{n+1}}(\varphi(t)) \geq T\left(F_{x_{0} x_{1}}(\varphi(t)-\varphi(c t)),\right. & \\
\left.F_{x_{1} x_{n+1}}(\varphi(c t))\right) \geq T\left(F_{x_{0} x_{1}}(\varphi(t)-\varphi(c t)),\right. & \\
\left.\min \left(F_{x_{0} x_{n}}(\varphi(t)), F_{x_{0} x_{1}}(\varphi(t)), F_{x_{n} x_{n+1}}(\varphi(t))\right)\right), & \forall t>0 .
\end{aligned}
$$

Given that

$$
\begin{array}{rlrl}
F_{x_{n} x_{n+1}}(\varphi(t)) & \geq F_{x_{0} x_{1}}\left(\varphi\left(\frac{t}{c^{n}}\right)\right) \geq F_{x_{0} x_{1}}(\varphi(t)), & \\
F_{x_{0} x_{1}}(\varphi(t)) & \geq F_{x_{0} x_{1}}(\varphi(t)-\varphi(c t)), \\
& \geq T^{n}\left(F_{x_{0} x_{1}}(\varphi(t)-\varphi(c t))\right), \quad \forall t>0,
\end{array}
$$

from the induction hypothesis we obtain

$$
\begin{aligned}
& F_{x_{0} x_{n+1}}(\varphi(t)) \geq T\left(F_{x_{0} x_{1}}(\varphi(t)-\varphi(c t)),\right. \\
& \left.T^{n}\left(F_{x_{0} x_{1}}(\varphi(t)-\varphi(c t))\right)\right) \\
& =T^{n+1}\left(F_{x_{0} x_{1}}(\varphi(t)-\varphi(c t))\right), \quad \forall t>0,
\end{aligned}
$$

which proves our claim.

Now, since $\lim _{t \rightarrow \infty} F_{x_{0} x_{1}}(\varphi(t)-\varphi(c t))=1$ and the family $\left\{T^{n}\right\}_{n}$ is equicontinuous at 1 , it follows that $D_{O(f, x)} \in \mathscr{D}^{+}$.
By setting $\varphi(t)=t$ in the above corollary we get the following.

Corollary 11. Let $(X, F, T)$ be a complete Menger space with $T$ being a continuous $t$-norm of Hadžić type and let $f: X \rightarrow X$ be a mapping such that

$$
\begin{aligned}
& F_{f(x) f(y)}(t) \\
& \quad \geq \min \left(F_{x y}\left(\frac{t}{c}\right), F_{x f(x)}\left(\frac{t}{c}\right), F_{y f(y)}\left(\frac{t}{c}\right)\right),
\end{aligned}
$$

for all $x, y \in X, t>0$. Then $f$ has a unique fixed point in $X$.

Remark 12. In paper [10], Babačev proved a fixed point result for mappings satisfying the contractive condition

$$
\begin{aligned}
& F_{f(x) f(y)}(\varphi(t)) \geq \min \left(F_{x y}\left(\varphi\left(\frac{t}{c}\right)\right),\right. \\
& F_{x f(x)}\left(\varphi\left(\frac{t}{c}\right)\right), F_{y f(y)}\left(\varphi\left(\frac{t}{c}\right)\right), F_{x f(y)}\left(2 \varphi\left(\frac{t}{c}\right)\right), \\
& \left.F_{y f(x)}\left(2 \varphi\left(\frac{t}{c}\right)\right)\right), \quad \forall t>0,
\end{aligned}
$$

for some altering distance function $\varphi$ and some $c \in(0,1)$, in Menger spaces with the $t$-norm $T_{M}$. We note that, by applying the triangle inequality,

$$
\begin{aligned}
& F_{x f(y)}\left(2 \varphi\left(\frac{t}{c}\right)\right) \\
& \geq \min \left(F_{x y}\left(\varphi\left(\frac{t}{c}\right)\right), F_{y f(y)}\left(\varphi\left(\frac{t}{c}\right)\right)\right), \\
& F_{y f(x)}\left(2 \varphi\left(\frac{t}{c}\right)\right) \\
& \geq \min \left(F_{x y}\left(\varphi\left(\frac{t}{c}\right)\right), F_{y f(y)}\left(\varphi\left(\frac{t}{c}\right)\right)\right),
\end{aligned}
$$

so this condition essentially reduces to (5). Therefore Theorem 9 improves the result in [10], as well as Ćirić's result in [7] (which can be obtained from that of Babačev for $\varphi(t)=$ $t)$. theorem.

Also, in [11], Choudhury et al. gave the following related

Theorem 13 (see [11]). Let $(X, F, T)$ be a complete Menger space with continuous $t$-norm $T$, and let $a, b, c$ be positive numbers with $a+b+c<1$, and $\varphi \in \Phi$. Suppose that $f: X \rightarrow X$ satisfies the inequality

$$
\begin{gathered}
F_{f(x) f(y)}(\varphi(t)) \geq \min \left(F_{x y}\left(\varphi\left(\frac{t_{1}}{a}\right)\right),\right. \\
\left.F_{x f(x)}\left(\varphi\left(\frac{t_{2}}{b}\right)\right), F_{y f(y)}\left(\varphi\left(\frac{t_{3}}{c}\right)\right)\right),
\end{gathered}
$$

for all $x, y \in X, t>0$, and $t_{1}, t_{2}, t_{3}>0$ with $t_{1}+t_{2}+t_{3}=t$. Then $f$ has a unique fixed point.

As indicated in [11], a mapping satisfying the contractive condition (29) must also verify our condition (5). Namely, 
suppose that (29) holds. Let $\varepsilon=(1-(a+b+c)) / 3 \in(0,1)$ and let $t_{1}=(a+\varepsilon) t, t_{2}=(b+\varepsilon) t, t_{3}=(c+\varepsilon) t$. It follows that

$$
\begin{aligned}
& F_{f(x) f(y)}(\varphi(t)) \geq \min \left(F_{x y}\left(\varphi\left(\frac{t(a+\varepsilon)}{a}\right)\right),\right. \\
&\left.F_{x f(x)}\left(\varphi\left(\frac{t(b+\varepsilon)}{b}\right)\right), F_{y f(y)}\left(\varphi\left(\frac{t(c+\varepsilon)}{c}\right)\right)\right), \\
& \forall t>0 .
\end{aligned}
$$

Due to the monotonicity of $\varphi$, the above relation implies that

$$
\begin{gathered}
F_{f(x) f(y)}(\varphi(t)) \geq \min \left(F_{x y}\left(\varphi\left(\frac{t}{k}\right)\right),\right. \\
\left.F_{x f(x)}\left(\varphi\left(\frac{t}{k}\right)\right), F_{y f(y)}\left(\varphi\left(\frac{t}{k}\right)\right)\right),
\end{gathered}
$$

for all $t>0$, where $k=\max (a /(a+\varepsilon), b /(b+\varepsilon), c /(c+\varepsilon)) \epsilon$ $(0,1)$.

Note that Theorem 13 only requires that the $t$-norm by which the space is endowed is continuous. Unfortunately, we can show that this assumption alone is not sufficient to guarantee the existence of fixed points for contractions of this type.

Specifically, let $\left(X, F, T_{L}\right)$ be a complete Menger space and let $f$ be a Sehgal contraction on $X$ with contraction constant $k<1 / 3$. Then

$$
\begin{aligned}
& F_{f(x) f(y)}(t) \geq F_{x y}\left(\frac{t}{k}\right) \\
& \geq \min \left(F_{x y}\left(\frac{t}{k}\right), F_{x f(x)}\left(\frac{t}{k}\right), F_{y f(y)}\left(\frac{t}{k}\right)\right) \\
& \geq \min \left(F_{x y}\left(\frac{t_{1}}{k}\right), F_{x f(x)}\left(\frac{t_{2}}{k}\right), F_{y f(y)}\left(\frac{t_{3}}{k}\right)\right),
\end{aligned}
$$

for all $x, y \in X, t>0$, and $t_{1}, t_{2}, t_{3}>0$ with $t_{1}+t_{2}+t_{3}=t$. Thus, $f$ satisfies the conditions of Theorem 13 with $a=b=$ $c=k$ and $\varphi(t)=t$. However, a well-known counterexample of Sherwood ([17], Corollary 1 of Theorem 3.5) shows that there exist Sehgal contractions on complete Menger spaces endowed with the $t$-norm $T_{L}$ having no fixed point.

It should be mentioned that a similar observation regarding continuity can be made with respect to Theorem 3.1 in [18], where the class of contractions considered also includes Sehgal contractions.

Finally, we illustrate the applicability of Theorem 9 with the following example.

Example 14. Let $X=[0,1]$ and $T(a, b)=a b$. Define $F_{x y}(t)=$ $(t /(t+1))^{|x-y|}$ for all $x, y \in X$ and $t>0 .(X, F, T)$ is a complete Menger space. We will only show that the triangle inequality is verified.
Assume that $t>s>0$ and $x, y, z \in X$. Since the function $t /(t+1)$ is increasing, it holds that

$$
\begin{aligned}
T\left(F_{x y}(t), F_{y z}(s)\right) & =\left(\frac{t}{t+1}\right)^{|x-y|}\left(\frac{s}{s+1}\right)^{|y-z|} \\
& \leq\left(\frac{t}{t+1}\right)^{|x-y|+|y-z|} \\
& \leq\left(\frac{t}{t+1}\right)^{|x-z|} \leq\left(\frac{t+s}{t+s+1}\right)^{|x-z|} \\
& =F_{x z}(t+s) .
\end{aligned}
$$

Let $\varphi(t)=t^{2} /(2 t+1)$ for all $t>0, c=1 / 2$, and

$$
f(x)= \begin{cases}\frac{x}{2}, & x \in[0,1), \\ 0, & x=1\end{cases}
$$

One can easily check that $\varphi$ is an altering distance function and that $O(f, x)$ is probabilistically bounded for every $x \in X$.

We will prove that condition (5) of Theorem 9 is satisfied. The following three cases are possible:

(1) If $x, y \in[0,1)$, then for all $t>0$ we have

$$
\begin{aligned}
F_{f(x) f(y)}(\varphi(t)) & =\left(\frac{t}{t+1}\right)^{2|f(x)-f(y)|}=\left(\frac{t}{t+1}\right)^{|x-y|} \\
& \geq\left(\frac{2 t}{2 t+1}\right)^{2|x-y|}=F_{x y}(\varphi(2 t)) .
\end{aligned}
$$

(2) If $x=y=1$, then

$$
F_{f(x) f(y)}(\varphi(t))=1=F_{x y}(\varphi(2 t))
$$

for all $t>0$.

(3) If $x \in[0,1)$ and $y=1$, then, for all $t>0$,

$$
\begin{aligned}
F_{f(x) f(y)}(\varphi(t)) & =\left(\frac{t}{t+1}\right)^{x} \geq \frac{t}{t+1} \geq\left(\frac{2 t}{2 t+1}\right)^{2} \\
& =\left(\frac{2 t}{2 t+1}\right)^{2|y-f(y)|}=F_{y f(y)}(\varphi(2 t))
\end{aligned}
$$

Thus the condition (5) is satisfied in this case as well.

However, note that by setting $x=2 / 3$ and $y=1$ we obtain

$$
\begin{aligned}
F_{f(x) f(y)}(\varphi(t)) & =\left(\frac{t}{t+1}\right)^{2 / 3}<\left(\frac{2 t}{2 t+1}\right)^{2 / 3} \\
& =F_{x y}(\varphi(2 t))
\end{aligned}
$$

for all $t>0$; therefore, $f$ does not satisfy the stronger condition (4).

By applying Theorem 9 we conclude that the function $f$ has a unique fixed point. It is easy to see that this point is $x=0$. 


\section{Conflict of Interests}

The authors declare that there is no conflict of interests regarding the publication of this paper.

\section{References}

[1] K. Menger, "Statistical metrics," Proceedings of the National Academy of Sciences of the United States of America, vol. 28, pp. 535-537, 1942.

[2] B. Schweizer and A. Sklar, Probabilistic Metric Spaces, NorthHolland Series in Probability and Applied Mathematics, NorthHolland Publishing, New York, NY, USA, 1983.

[3] G. Constantin and I. Istrăţescu, Elements of Probabilistic Analysis with Applications, Kluwer Academic Publishers, 1989.

[4] S. Romaguera, A. Sapena, and P. Tirado, "The Banach fixed point theorem in fuzzy quasi-metric spaces with application to the domain of words," Topology and Its Applications, vol. 154, no. 10, pp. 2196-2203, 2007.

[5] S. Romaguera and P. Tirado, "The complexity probabilistic quasi-metric space," Journal of Mathematical Analysis and Applications, vol. 376, no. 2, pp. 732-740, 2011.

[6] J. Sun, X. Wu, V. Palade, W. Fang, C.-H. Lai, and W. Xu, "Convergence analysis and improvements of quantum-behaved particle swarm optimization," Information Sciences, vol. 193, pp. 81-103, 2012.

[7] L. B. Ćirić, "On fixed points of generalized contractions on probabilistic metric spaces," Publications de l'Institut Mathématique (Beograd), vol. 18, no. 32, pp. 71-78, 1975.

[8] B. S. Choudhury and K. Das, "A new contraction principle in Menger spaces," Acta Mathematica Sinica, vol. 24, no. 8, pp. 1379-1386, 2008.

[9] D. Miheț, "Altering distances in probabilistic Menger spaces," Nonlinear Analysis: Theory, Methods \& Applications, vol. 71, no. 7-8, pp. 2734-2738, 2009.

[10] N. A. Babačev, "Nonlinear generalized contractions on Menger PM spaces," Applicable Analysis and Discrete Mathematics, vol. 6, no. 2, pp. 257-264, 2012.

[11] B. S. Choudhury, K. Das, and P. N. Dutta, "A fixed point result in Menger spaces using a real function," Acta Mathematica Hungarica, vol. 122, no. 3, pp. 203-216, 2009.

[12] O. Hadžić, "On the $(\varepsilon, \lambda)$-topology of probabilistic locally convex spaces," Glasnik Matematicki. Serija III, vol. 13, no. 33, 2, pp. 293-297, 1978.

[13] V. M. Sehgal, Some fixed point theorems in functional analysis and probability [Ph.D. thesis], Wayne State University, 1966.

[14] O. Hadžić and E. Pap, Fixed Point Theory in Probabilistic Metric Spaces, Kluwer Academic Publishers, 2001.

[15] V. Radu, "On the t-norms of Hadžić type and fixed points in PM-spaces," Review of Research (Novi Sad), vol. 13, pp. 81-86, 1983.

[16] M. Khan, M. Swaleh, and S. Sessa, "Fixed point theorems by altering distances between the points," Bulletin of the Australian Mathematical Society, vol. 30, no. 1, pp. 1-9, 1984.

[17] H. Sherwood, "Complete probabilistic metric spaces," Zeitschrift für Wahrscheinlichkeitstheorie und Verwandte Gebiete, vol. 20, no. 2, pp. 117-128, 1971.

[18] T. Došenović, P. Kumam, D. Gopal, D. K. Patel, and A. Takači, "On fixed point theorems involving altering distances in Menger probabilistic metric spaces," Journal of Inequalities and Applications, vol. 2013, article 576, 10 pages, 2013. 


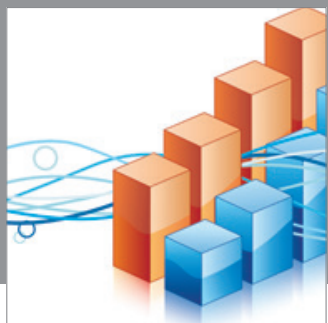

Advances in

Operations Research

mansans

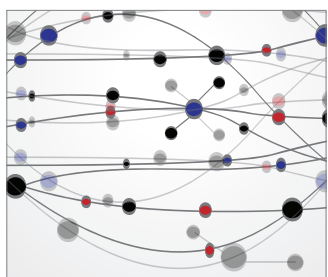

The Scientific World Journal
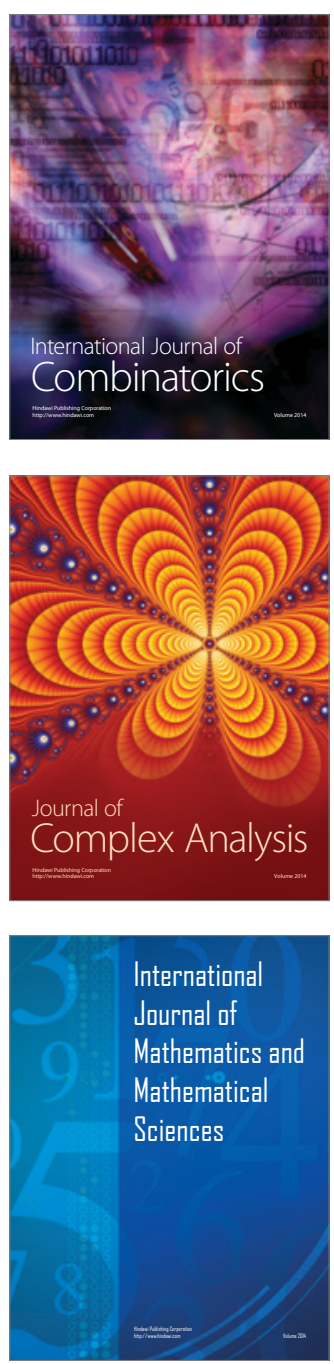
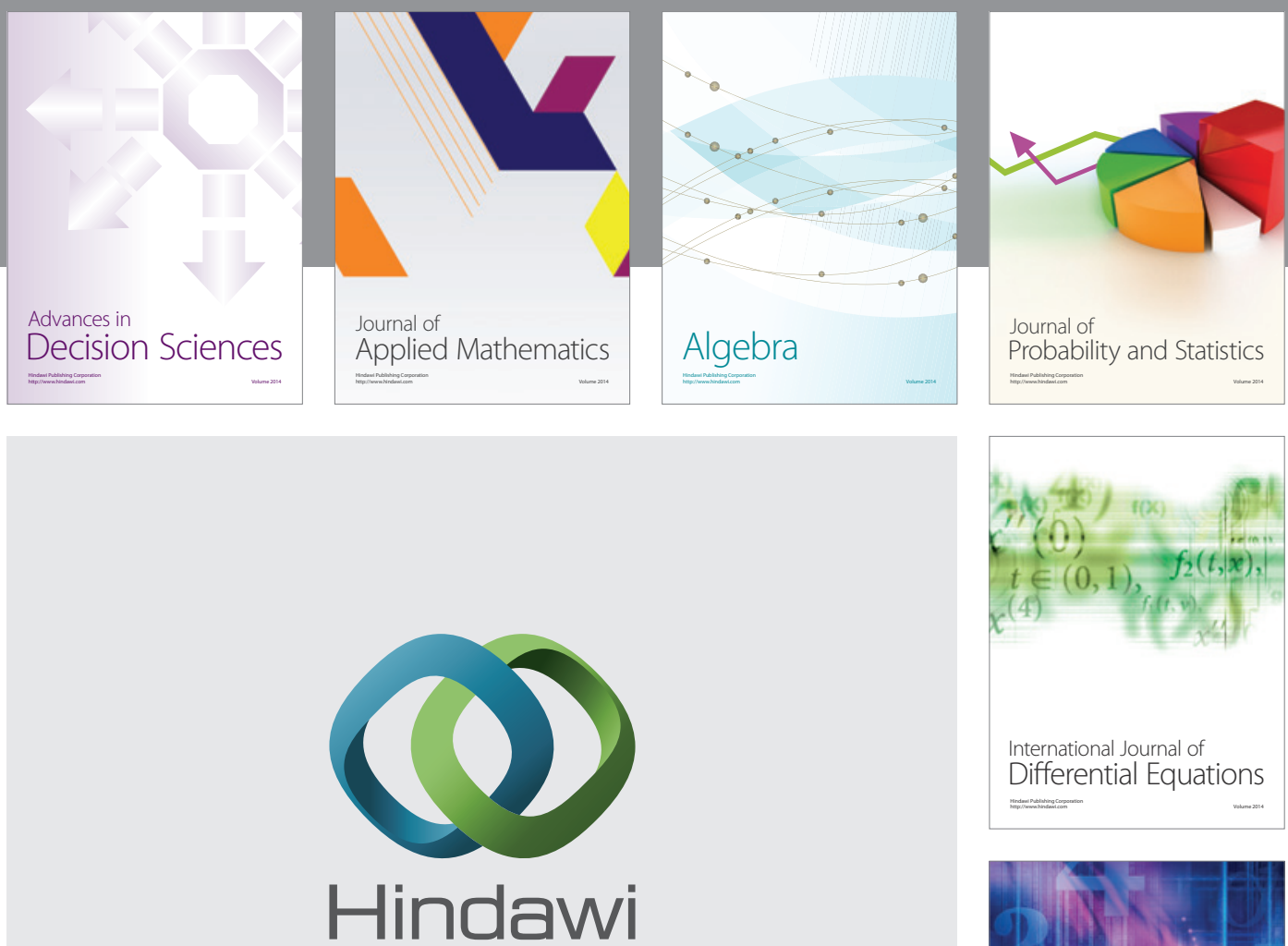

Submit your manuscripts at http://www.hindawi.com
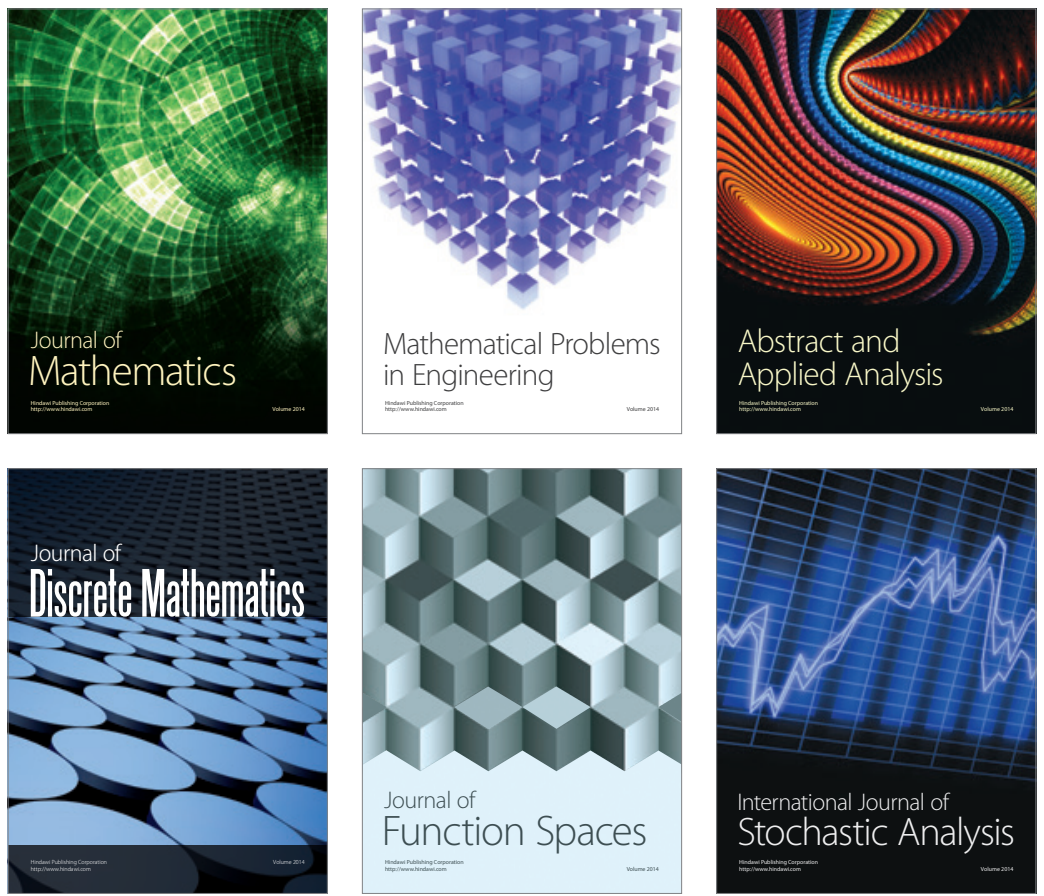

Journal of

Function Spaces

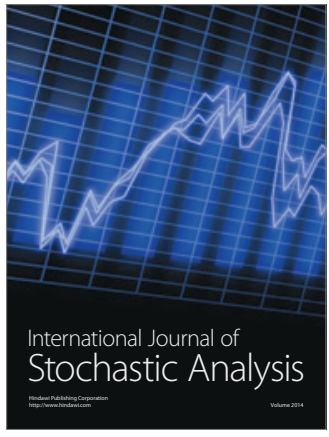

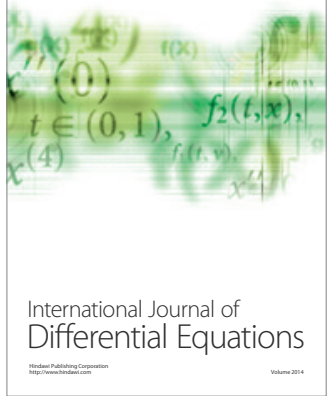
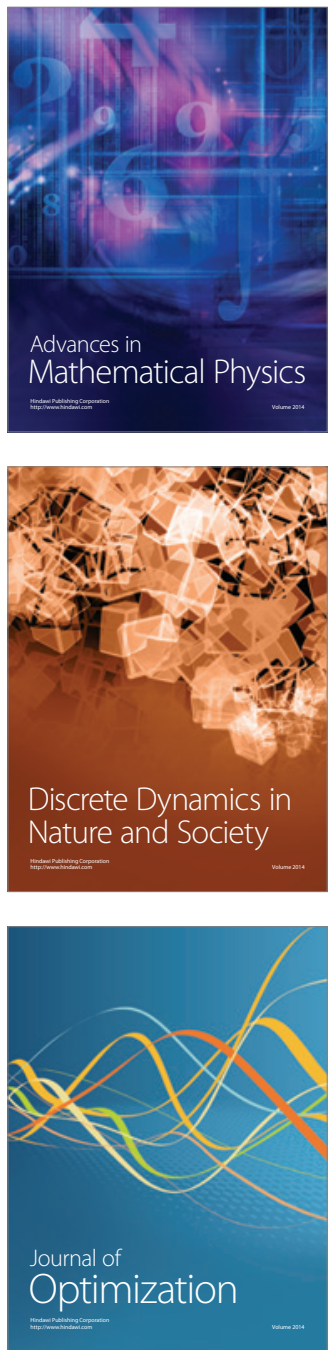